\title{
High-precision bio-replication of synthetic drag reduction shark skin
}

\author{
ZHANG DeYuan $^{1 *}$, LI YuanYue ${ }^{1}$, HAN Xin $^{2}$, LI Xiang ${ }^{1} \&$ CHEN HuaWei ${ }^{1}$ \\ ${ }^{1}$ Bionic and Micro/Nano/Bio Manufacturing Technology Research Center, Beihang University, Beijing 100191, China; \\ ${ }^{2}$ School of Agricultural and Food Engineering, Shandong University of Technology, Zibo 255049, China
}

Received April 20, 2010; accepted August 11, 2010; published online January 17, 2011

\begin{abstract}
Nano-long chains were grafted over the replicated micro-grooves of shark skin in a novel attempt to replicate bio-synthetic drag reduction structure with high precision through synthetic bio-replication. Pre-treated shark skin was used as casting template to prepare a flexible female die of silicone rubber by soft die formation. A waterborne epoxy resin was then used to graft long-chains of drag reduction agent and prepare a synthetic drag reduction shark skin with nano-long chain drag reduction interface and lifelike micro-grooves. Replication precision analysis shows that this technology could replicate the complicated three-dimensional morphology of a biological drag reduction surface with high precision. Drag reduction experiments show that the material had an excellent synthetic drag reduction effect, with a maximal drag reduction rate of up to $24.6 \%$ over the velocities tested.
\end{abstract}

synthetic drag reduction, high-precision, shark skin, synthetic bio-replication, micro-groove, nano-long chain

Citation: Zhang D Y, Li Y Y, Han X, et al. High-precision bio-replication of synthetic drag reduction shark skin. Chinese Sci Bull, 2011, 56: 938-944, doi: $10.1007 / \mathrm{s} 11434-010-4163-7$

The morphology of shark skin could be replicated initially by bio-replicated forming, such as micro-embossing or micro-molding. To enable the replication material to fill in the concave surfaces of shark skin, a pressing step must be added in both of these approaches, which causes an increase in groove width, a reduction in ridge height and a distortion in scale obliquity [1]. These hamper the achievement of high replication precision. The drag reduction rate of bio-replicated shark skin has reached only $8.25 \%$ [2], below the bottleneck of $10 \%$ for biomimetic micro-groove structure [3] and far below the performance of natural shark skin. Natural shark skin has excellent drag reduction performance because of a hierarchical structure built up by microgrooves and nano-long chain mucus drag reduction interface around the shark body [4]. Its drag reduction performance is difficult to compare with single groove structure. Therefore, replicate synthetic drag reduction shark skin with both micro-grooves and nano-long chain drag reduction interface is increasingly sought after.

Synthetic drag reduction has been explored over the past

*Corresponding author (email: zhangdy@buaa.edu.cn) decades, but its bio-drag reduction morphology was simplified and bio-drag reduction interface was formed by injecting drag reduction agent (DRA) or coating polymer, which has serious problems, such as the morphology of micro-grooves being different from the biology and the waste of DRA [5-10]. Therefore, a novel synthetic bio-replication forming approach is proposed in this paper for simultaneously grafting nano-long chains to bio-replicated micro-grooves of shark skin, which maintains the simplicity of bio-replicated forming. Synthetic bio-replication combines soft die forming and grafting technology, which has advantages, such as resulting in high replication precision and saving of DRA. It provides an effective approach for the manufacture of synthetic drag reduction shark skin with high replication precision and excellent drag reduction performance.

Soft lithography is a recent approach to manufacture nano/micro structures that can precisely replicate nano/micro hierarchical biological surface structures, such as those of the lotus and diatom, using UV-curable resins and nanoimprint lithography [11-13]. Graft copolymerization improves the performance of polymeric materials by binding appropriate branched chains or functional lateral groups 
to the macromolecule chain through chemical bonds [14]. Synthetic bio-replication combines soft lithography and graft copolymerization, which could realize the grafting of nano-long chains simultaneously over the replicated micro-grooves of shark skin with high precision. This is a novel approach to manufacture bio-drag reduction structures.

\section{Materials and methods}

\subsection{Materials}

Shark skin $(150 \mathrm{~mm} \times 150 \mathrm{~mm})$ of Isurus oxyrinchus (body side), which is a typically fast-swimming shark, was taken as the template for bio-replication [15]. A condensation bi-component silicone rubber RTV-2 875 (Room Temperature Vulcanized), which has two components, is an excellent silicone rubber to use for dies because of its advantages such as curing uniform between surface and interior, stressfree after curing, heat shrinkage is low $(0.2 \%)$, and excellent demolding performance. It could be applied to manufacture a flexible female die [16]. A waterborne epoxy resin, composed of water-based epoxy resin emulsion (AB-EP-44) and waterborne epoxy curing agent (AB-HGF), was used for forming the replicate material base. Polyacrylamide (PAM), a water-soluble linear polymer drag reduction agent with a molecular weight of over 3 million and a chain segment length of $0.25 \mathrm{~nm}$ [17], was used for forming the nano-long chain drag reduction interface. A graft copolymer of waterborne epoxy resin and PAM was used as the substrate of synthetic drag reduction shark skin. Dibutyl phthalate (DBP), a universal plasticizer [18], was used for improving the ductility and flexibility of substrate.

\subsection{Synthetic bio-replication of shark skin}

Figure 1 shows the process for synthetic bio-replication of shark skin in four steps: pre-treatment of the shark skin, molding of the flexible female die, preparation of the prepolymer and formation of the synthetic drag reduction shark skin.

(i) Pre-treatment of the shark skin. Pre-treatment of the shark skin is of great importance in maintaining its micro-groove structure and improving its strength as the molding template. Here, the pre-treatment of the shark skin followed six steps: rigidity fixing, cleaning, chemical fixing, rinsing, dehydration and drying. To prevent the shark skin from shrinking and deforming during pre-treatment, it was fixed on a rigid plate with nails or clamps before cleaning. Figure 2(a) shows the micro-grooves of shark skin after pre-treatment.

(ii) Molding of the flexible female die. A flexible, silicone rubber, female die was made by soft die forming technology using in soft lithography. A given amount of silicone rubber was first weighed out. The mass of components $\mathrm{A}$ and $\mathrm{B}$ of the silicone rubber compound were determined on the basis of desired thickness and area of the flexible female die. The components were mixed well and then degassed under vacuum. Vacuum degassing was conducted again after pouring the mixture onto the shark skin to remove air from the cavities of the shark skin surface. Finally, the silicon rubber die was cured and then demolded at room temperature. Figure 2(b) shows a scanning electron micrograph of the flexible female die.

(iii) Formation of the synthetic drag reduction shark skin. A graft copolymer of water-based epoxy resin and PAM was used as the substrate of the synthetic drag reduction shark skin. A PAM solution of given concentration was first prepared and the water-based epoxy resin emulsion was then added and mixed for several minutes. Curing agent and plasticizer were added and stirred for a few minutes and then filtered and degassed. Vacuum degassing was conducted again after pouring the prepolymer onto the surface of the female die. The material was then placed inside an
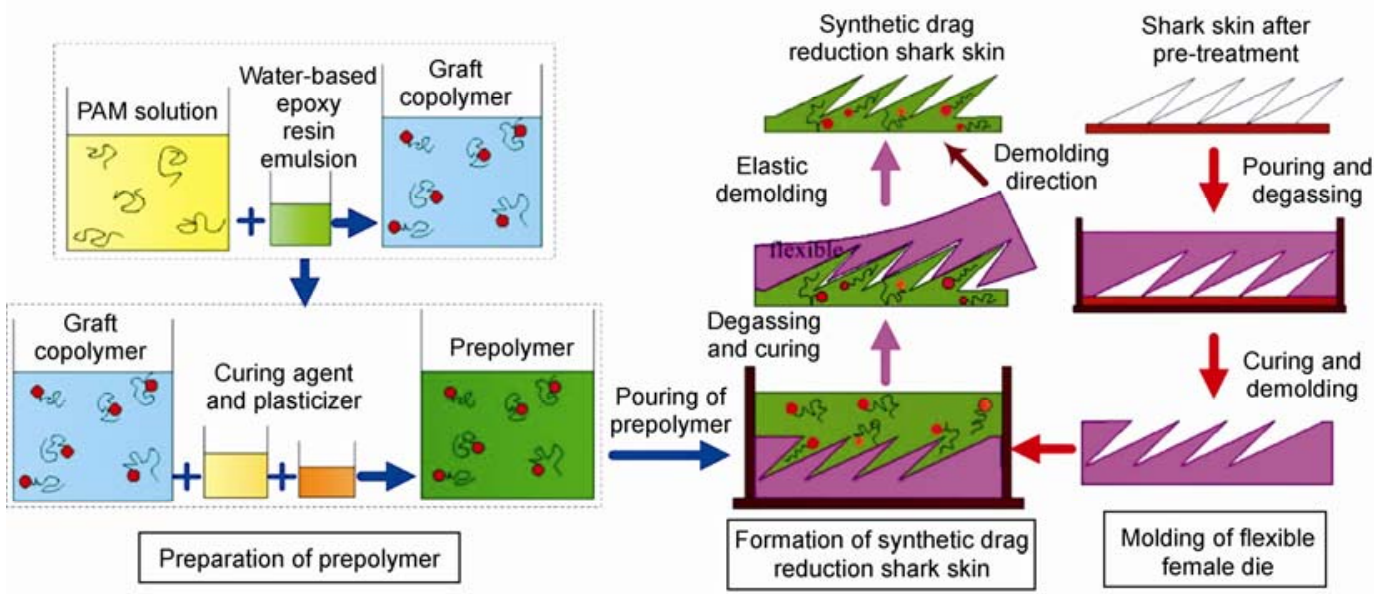

Figure 1 Synthetic bio-replication of shark skin process. 

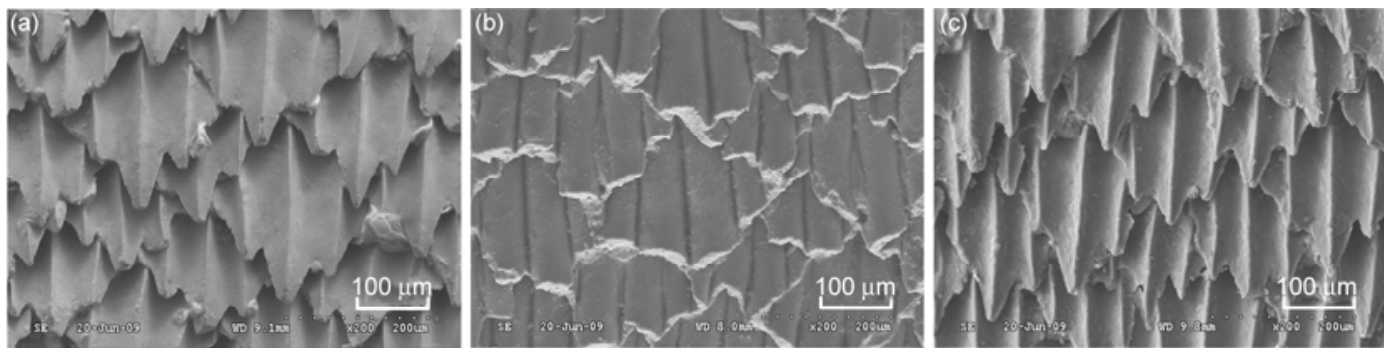

Figure 2 Scanning electron micrograph of (a) shark skin, (b) flexible silicone rubber female die and (c) synthetic drag reduction shark skin.

electric blast drying oven and cured. After curing, the synthetic drag reduction shark skin was obtained by flexible demolding. Figure 2(c) shows a SEM image of the synthetic shark skin.

\section{Results and discussions}

\subsection{Analysis of replication precision}

The horizontal section curve on central section is important for determining the shape of the shark scale and the vertical section curve along the central ridge is important for determining the obliquity of the shark scale. This paper analyzes the replication precision of synthetic drag reduction shark skin by comparing its three-dimensional scanned photo with shark skin. The three-dimensional scan photos, shown in Figure 3, were taken using a three-dimensional non-touch white light interference topography instrument. The curves of horizontal and vertical sections on corresponding positions of the samples were obtained by SPIP software, and these positions are shown as white lines in Figure 3. Figure 4 and Figure 5 show the curves of the horizontal and vertical sections.
Ideally, one should select the scale with its exact corresponding replication scale, but it is difficult in practice. Considering that the shape of scales on shark skin depend on their positions and the species of shark, we marked scales on the shark skin first and then prepared the corresponding synthetic drag reduction shark skin and compared the scales in a corresponding position around the mark point to analyze the replication precision. Although we could not find out the exact corresponding scales, the two scales we selected were the same in structure, so it appears to be a technically feasible approach.

Figure 4 shows the horizontal section curve on central section. The deformation rate of the central ridge height and the left/right groove width are shown in Table 1. Figure 5 shows the vertical section curve along the central ridge. By calculating the height offset along the direction of the groove every other $10 \mu \mathrm{m}$, the average height offset rate of the vertical section curve was obtained. The results are shown in Table 1.

From Table 1, we can see that the replication precision of the height of horizontal section, the width of left and right grooves and the obliquity of scale on synthetic drag reduction shark skin formed by synthetic bio-replication were
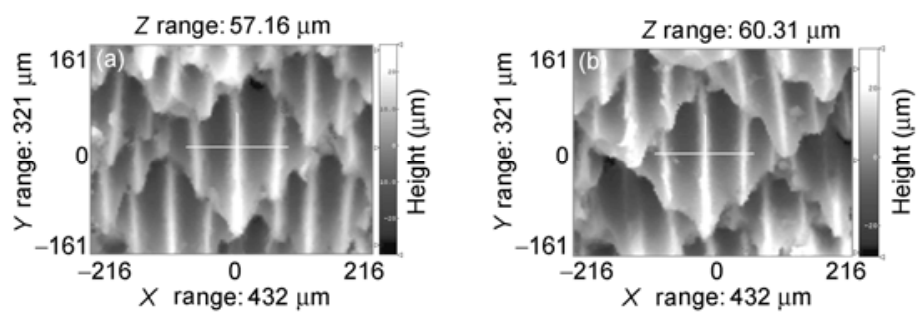

Figure 3 Scanning photos of (a) shark skin sample and (b) the corresponding synthetic drag reduction shark skin.

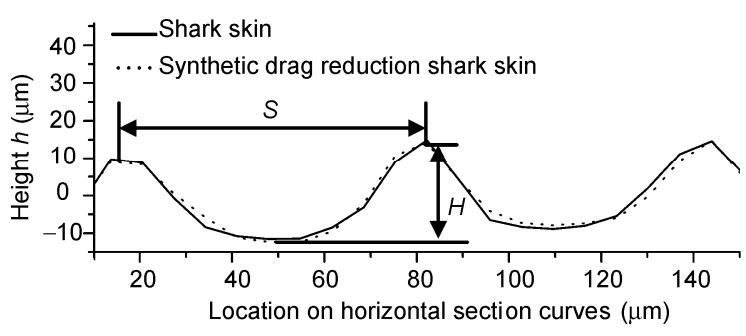

Figure 4 Horizontal section curve on the central section.

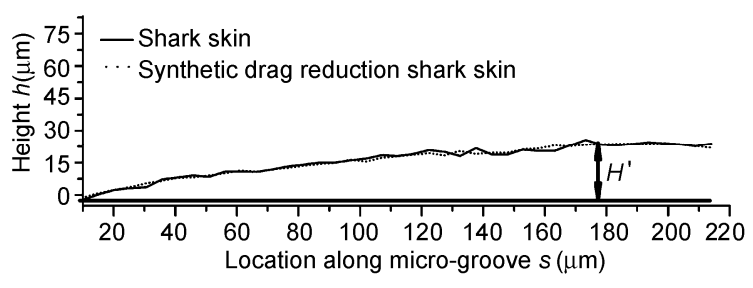

Figure 5 Vertical section curve along the central ridge. 
Table 1 Analysis of replication precision of horizontal and vertical section curves ${ }^{\text {a) }}$

\begin{tabular}{|c|c|c|c|c|c|c|c|c|}
\hline & \multicolumn{2}{|c|}{ Vertical section } & \multicolumn{6}{|c|}{ Cross section } \\
\hline & \multirow{2}{*}{$\begin{array}{l}\text { Average height } \\
\text { offset } \Delta H^{\prime}(\mu \mathrm{m})\end{array}$} & \multirow{2}{*}{$\begin{array}{l}\text { Average height } \\
\text { offset rate }(\%)\end{array}$} & \multirow{2}{*}{$\begin{array}{l}\text { Central ridge } \\
\text { height } H(\mu \mathrm{m})\end{array}$} & \multirow{2}{*}{$\begin{array}{l}\text { Deformation rate of } \\
\text { Central ridge height } \\
\qquad(\%)\end{array}$} & \multicolumn{2}{|c|}{ Width of groove $S(\mu \mathrm{m})$} & \multicolumn{2}{|c|}{$\begin{array}{l}\text { Deformation rate of } \\
\text { groove width }(\%)\end{array}$} \\
\hline & & & & & Left side & Right side & Left side & Right side \\
\hline $\begin{array}{c}\text { Synthetic drag reduction } \\
\text { shark skin }\end{array}$ & 0.98 & $3.7 \%$ & 26.2 & -2.6 & 68.36 & 61.41 & -0.1 & $-0.4 \%$ \\
\hline
\end{tabular}

a) $H_{\max }{ }^{\prime}=26.7 \mu \mathrm{m}$.

remarkably improved over previous attempts by microembossing and micro-molding [1].

The vertical section curves along central ridge of bioshark skin manufactured by micro-embossing and micromolding deviated significantly from the shark skin sample. Their height offset increased from the middle part and reached a maximum at the end. Their deformation rates were $27.5 \%$ and $13.7 \%$ respectively. However, from Figure 5 we can see that the vertical section curve of synthetic drag reduction shark skin manufactured by synthetic bio-replication is almost coincident with the shark skin sample, with a height offset randomly distributed and an average deformation rate of only $3.7 \%$.

The replication precision is related to the scale structure and technical process. Figure 6 shows the distortion process of scale obliquity during micro-embossing and micro-molding. Scales on the shark skin are composed of base plate and spine. The base plate is embedded in skin and the spine protrudes outside, which creates a cantilever beam structure. The replication materials used for female dies during micro-embossing and micro-molding do not possess good fluidity. In order to improve the fluidity of replication materials to make them fill in the cavities of shark skin surface, they must be pressed and heated during micro-embossing and micro-molding. These technical processes cause bending of the spine. The closer to the end of scale, the more deformation occurs. Consequently, the scale obliquity is seriously distorted in the final product. On the other hand, the replication material used for flexible female die during synthetic bio-replication is silicon rubber, which has excellent fluidity. Silicon rubber is capable of filling in the cavities without heating and pressing, which guarantees the high replication precision of geometrical morphology and maintains the scales obliquity.

Furthermore, the graft copolymer of waterborne epoxy resin and PAM was used as the substrate of synthetic drag reduction shark skin. The substrate without additive is crumbly and hard to demolding after curing. To overcome these problems, plasticizer is added to the substrate. Figure 7 shows the influence of plasticizer addition to performance of the substrate, where the proportion of plasticizer was increased from sample 1 (no plasticizer) to sample 7. From Figure 7, we can see that the drawing deformation rate of substrate was the maximum, its elasticity modulus was nearly the lowest, its flexibility was good and its integral performance was optimal for sample 4 . The excellent fluidity

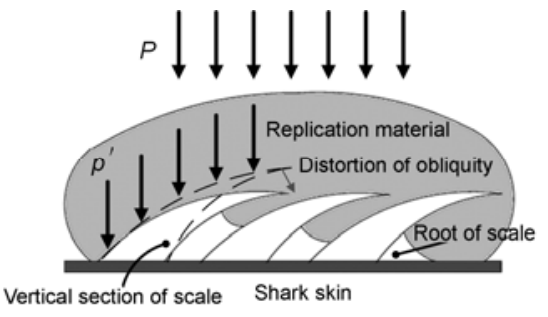

Figure 6 Distortion process of scale obliquity.

of substrate with plasticizer and the ease of demolding after curing enabled the replication of flexible female die with high precision. For these reasons, the synthetic bio-replication technology has a higher replication precision than micro-embossing and micro-molding.

\subsection{Validation of the synthetic drag reduction effect}

(i) Structural model of the synthetic drag reduction shark skin. The mechanism of grafting is that some of the amide groups of PAM react with the epoxy groups of water-based epoxy resin, which leads to the ring-opening reaction of epoxy groups for generation of hydroxyl groups, as shown in Figure 8 [19]. Figure 9 shows the structural model of the synthetic drag reduction shark skin. When it is placed in turbulent flow, the micro-grooves could reduce energy consumption induced by longitudinal vortices [20]. The long-chains of PAM, with abundant hydrophilic groups extending into the buffer layer, could inhibit the radial pulsation

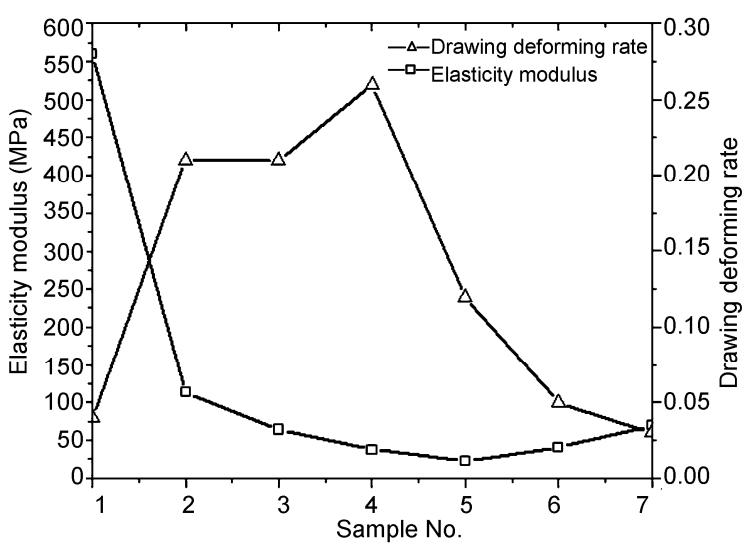

Figure 7 Influence of increasing amounts of plasticizer from sample 1 to 7 on the performance of substrate. 


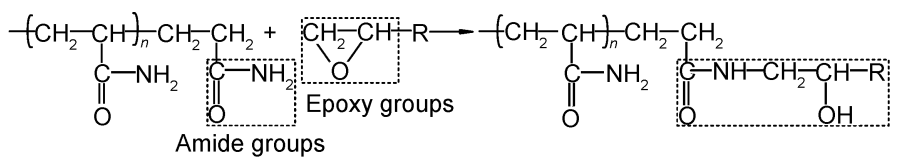

Figure 8 Grafting mechanism of water-based epoxy resin and PAM.

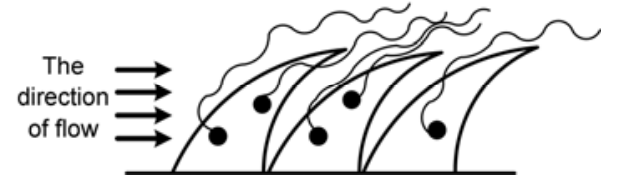

Figure 9 Structural model of synthetic drag reduction shark skin.

of the fluid micella to decrease energy consumption [21]. As a result of such synthetic effect, the drag reduction was remarkably improved.

(ii) Preparation of samples and the test process. To validate the synthetic drag reduction effect of synthetic drag reduction shark skin, various experiments were conducted in a vacuole water tunnel at the China Ship Scientific Research Center (CSSRC). The length of the test section was $3.2 \mathrm{~m}$ and the diameter was $0.8 \mathrm{~m}$. The flow velocity in the test section was adjustable between 0 and $20 \mathrm{~m} / \mathrm{s}$. The measurement range of the strain gauge balances was $300 \mathrm{~N}$ and its resolution was $0.1 \mathrm{~N}$.

Two test models with a hollow elliptical sphere at the front end and a hollow cylinder at the posterior segment were made from aluminum alloy LY12. The size of each test model, with the length $(500 \mathrm{~mm})$ and external diameter (90 $\mathrm{mm}$ ) of the cylindrical section were determined according to the size of the strain gauge balances. The external diameters of the hollow elliptical spheres were $94 \mathrm{~mm}$ for model 1 and $90.6 \mathrm{~mm}$ for model 2, to guarantee the outside surfaces would be smooth after pasting test skins of different depth.

The test skins were divided into two groups. The first group, including the smooth skin (G-1) and the bio-shark skin micro-groove drag reduction skin (G-2), were made from silicone rubber, with sizes $170 \mathrm{~mm} \times 97 \mathrm{~mm} \times 2 \mathrm{~mm}$ (length $\times$ width $\times$ height). They were used for testing the drag reduction performance of the micro-groove structure and were pasted upon the surface of test model 1 . The second group were made from waterborne epoxy resin, including the smooth skin (H-1), the nano-long chain skin (H-2) and the synthetic drag reduction shark skin (H-3). The drag reduction performances of nano-long chain skin and synthetic drag reduction shark skin were measured by experiments on the second group. Test skins of the second group with size $170 \mathrm{~mm} \times 97 \mathrm{~mm} \times 0.3 \mathrm{~mm}$ (length $\times$ width $\times$ height $)$ were pasted to test model 2.

The installation of test models was divided into three parts: installation of the test models and balance; the balance and supporting part; and the test section in the water tunnel. Figure 10 shows the test section in the system in which the balance is covered with a guided flow dome to reduce additional resistance. The balance and supporting part were not disassembled during the test process and the test models, connecting to the balance by a thread, were the only parts replaced.

The experiments were conducted according to the rules for the vacuole water tunnel test (Q/702J0301-2008). The water temperature was set $28^{\circ} \mathrm{C}$ and the test system was degassed for more than $1 \mathrm{~h}$ before testing. The flow velocity began at $3.3 \mathrm{~m} / \mathrm{s}$ and continuously increased until the test skins were washed off.

(3) The test results. The resistance values of groups 1 and 2 are listed in Table 2. The sum of drag reduction rate of micro-groove and nano-long chain skins, DR1, and the drag reduction rate of synthetic drag reduction shark skin, DR2, are calculated on the basis of the resistance values and are shown in Table 2. The drag reduction rate curves of DR1 and DR2 are shown in Figure 11. DR3 is the drag reduction rate curve of the bio-shark skin micro-groove drag reduction skin manufactured by micro-embossing.

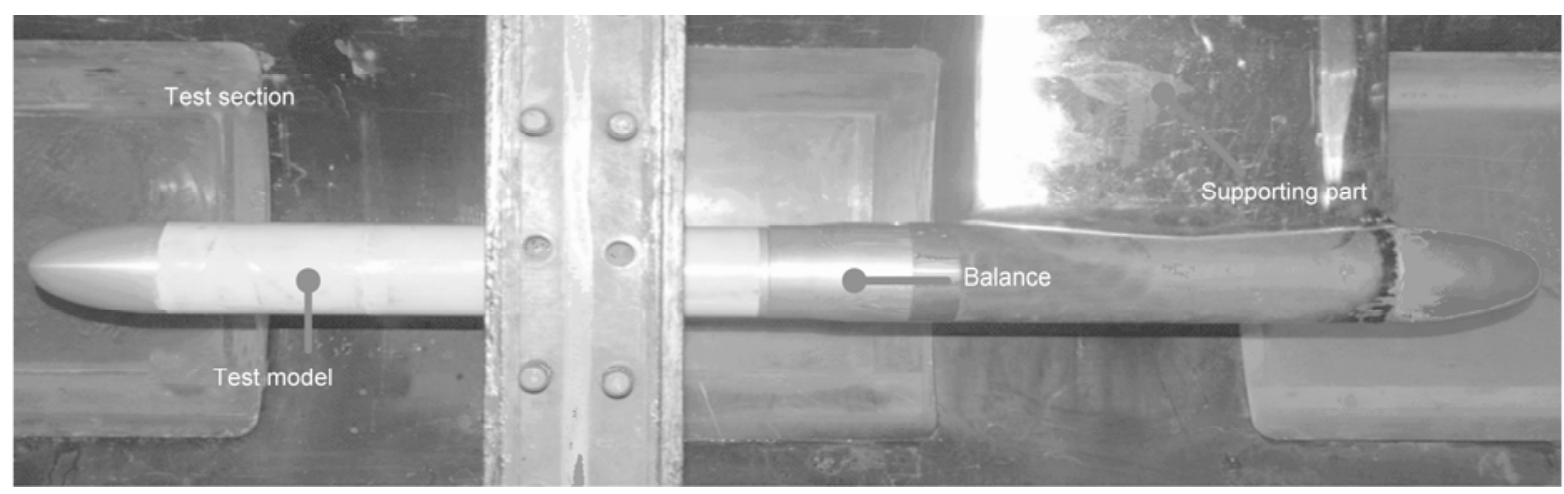

Figure 10 Test model and its installation. 
Table 2 Resistance values and drag reduction rates

\begin{tabular}{|c|c|c|c|c|c|c|c|}
\hline \multirow{2}{*}{$\begin{array}{c}\text { Flow velocity } \\
\qquad v\left(\mathrm{~m} \mathrm{~s}^{-1}\right)\end{array}$} & \multicolumn{5}{|c|}{ Resistance values $F(\mathrm{~N})$} & \multicolumn{2}{|c|}{ Drag reduction rate DR $(\%)$} \\
\hline & $\mathrm{G}-1 / F_{1}$ & $\mathrm{G}-2 / F_{2}$ & $\mathrm{H}-1 / F_{1}{ }^{\prime}$ & $\mathrm{H}-2 / F_{2}^{\prime}$ & $\mathrm{H}-3 / F_{3}{ }^{\prime}$ & $(\mathrm{H}-2)+(\mathrm{G}-2) / \mathrm{DR}_{1}$ & $\mathrm{H}-3 / \mathrm{DR}_{2}$ \\
\hline 3.3 & 2.4 & 2.1 & 1.5 & 1.5 & 1.4 & 13.5 & 6 \\
\hline 3.6 & 3.1 & 2.8 & 2.4 & 2.2 & 2.1 & 18.1 & 10.6 \\
\hline 3.9 & 3.9 & 3.5 & 3.3 & 3.0 & 2.8 & 19.2 & 13.2 \\
\hline 4.2 & 4.9 & 4.3 & 4.2 & 3.8 & 3.6 & 19.8 & 15.0 \\
\hline 4.5 & 5.8 & 5.2 & 5.2 & 4.7 & 4.4 & 20.1 & 16.3 \\
\hline 5 & 7.6 & 6.9 & 7.0 & 6.2 & 5.7 & 20.1 & 18.2 \\
\hline 5.5 & 9.8 & 8.8 & 8.9 & 8.0 & 7.2 & 19.7 & 19.6 \\
\hline 6 & 12.2 & 11 & 11.0 & 9.9 & 8.7 & 19.5 & 20.9 \\
\hline 6.5 & 14.8 & 13.4 & 13.2 & 11.9 & 10.3 & 19.3 & 22.0 \\
\hline 7 & - & - & 15.5 & 14.1 & 11.9 & - & 22.9 \\
\hline 7.5 & - & - & 17.9 & 16.5 & 13.7 & - & 23.8 \\
\hline 8 & - & - & 20.6 & 19.0 & 15.5 & - & 24.6 \\
\hline
\end{tabular}

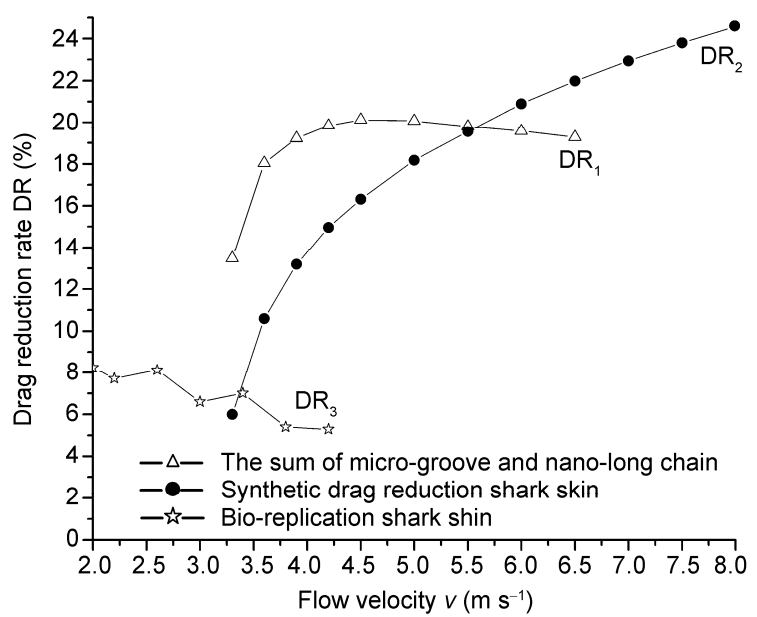

Figure 11 Drag reduction rate curve.

From Figure 11 we can see that the drag reduction rate of synthetic drag reduction shark skin increases steadily with the increase of flow velocity. When the flow velocity was between $3.3 \mathrm{~m} / \mathrm{s}$ and $5.5 \mathrm{~m} / \mathrm{s}$, the drag reduction rate of synthetic drag reduction shark skin, DR2, was less than the sum of the micro-groove and nano-long chain, DR1. When the flow velocity exceeded $5.5 \mathrm{~m} / \mathrm{s}$, DR2 steadily increased and exceeded DR1, which already shown a downward trend. The synthetic drag reduction effect then began to appear. When the test was terminated at a flow velocity of up to $8 \mathrm{~m} / \mathrm{s}$, DR2 reaches to the maximum, $24.6 \%$, which was much higher than the maximal drag reduction rate, $8.25 \%$, of the single bio-shark skin micro-groove drag reduction skin (DR3). Therefore, the synthetic bio-replication technology enabled both the synthetic drag reduction of micro-grooves and nano-long chains and remarkably improved the overall drag reduction effect.

\section{Conclusions}

(1) The analysis of replication precision indicates that syn- thetic bio-replication can realize the replication of shark skin morphology with high precision. The replication of scale obliquity, with an average deformation rate of $3.7 \%$, in particular, was remarkably improved over micro-embossing and micro-molding.

(2) The drag reduction performance test indicates that the synthetic drag reduction shark skin using the graft copolymer of waterborne epoxy resin and PAM as the substrate could enable drag reduction by lifelike micro-grooves and nano-long chains. It has a remarkable synthetic drag reduction effect, reaching up to $24.6 \%$ during the test process.

This paper replicated the complicated three-dimensional morphology of shark skin with high precision and realized the synthetic drag reduction effect of micro-grooves and nano-long chains. However, the structural model of synthetic drag reduction shark skin needs to be confirmed and the synthetic drag reduction behaviors under higher flow velocities require further experiment research.

The authors express their great thanks to the China Ship Scientific Research Center for providing experimental support. This work was supported by the National Natural Science Foundation of China (50775006), the National Defense Basic Science Research Special Foundation (D2120060002) and the Key Project of National High-Tech R\&D Program of China (2009AA043802).

1 Han X, Zhang D Y. Study on the micro-replication of shark skin. Sci China Ser E: Technol Sci, 2008, 51: 890-896

2 Han X, Zhang D Y, Li X, et al. Bio-replicated forming of the biomimetic drag-reducing surfaces in large area based on shark skin. Chinese Sci Bull, 2008, 53: 1587-1592

3 Bechert D W, Bruse M, Hage W, et al. Experiments on drag-reducing surfaces and their optimization with an adjustable geometry. J Fluid Mech, 1997, 338: 59-87

4 Bechert D W, Hoppe G, Reif W E. On the drag reduction of the shark skin. In: Davis S H, Lumley J L, eds. AIAA Shear Flow Control Conference, 1985 Mar 12-14, Boulder, Colorado. Berlin: Springer-Verlag Press, 1985. 1-17

5 Choi K S, Gadd G E, Pearcey H H, et al. Tests of drag-reducing polymer coated on a riblet surface. Appl Sci Res, 1989, 46: 209-216

6 Rohr J, Anderson G W, Reidy L W. An experimental investigation of the drag reducing effects of riblet in pipes. In: Sellin R H J, Moses R 
T, eds. Drag Reduction in Fluid Flows: Techniques for Friction Control. Chichester: Ellis Horwood Limited Publishers, 1990. 263-270

7 Christodoulou C, Liu K N, Joseph D D. Combined effects of riblets and polymers on drag reduction in pipes. Phys Fluids A, 1991, 3: 995-996

8 Anderson G W, Rohr J J, Stanley S D. The combined drag effects of riblets and polymers in pipe flow. J Fluids Eng, 1993, 115: 213-221

9 Koury E, Virk P S. Drag reduction by polymer solutions in a riblet-lined pipe. Appl Sci Res, 1995, 54: 323-347

10 Mizunuma H, Ueda K, Yokouchi Y. Synergistic effects in turbulent drag reduction by riblets and polymer additives. J Fluids Eng, 1999, 121: 533-540

11 Dusan L, James G M, Ratnesh L, et al. Rapid fabrication of microand nanoscale patterns by replica molding from diatom biosilica. Adv Funct Mater, 2007, 17: 2439-2446

12 Liu B, He Y N, Fan Y, et al. Fabricating super-hydrophobic lotus-leaf-like surfaces through soft-lithographic imprinting. Macromol Rapid Comm, 2006, 27: 1859-1864

13 Liu B, Fu Y Q, Ruan W Q, et al. Preparation of super-hydrophobic surfaces by using elastomer templates and UV-curable resin. Acta
Polym Sin, 2008, 2: 155-160

14 Wang C, Yan Y R. Modification of Polymer Materials (in Chinese). Beijing: China Textiles Press, 2007. 7-12

15 Zhu Y D, Meng Q W. Fauna S, Cyclosyomata, Chondrichthyes (in Chinese). Beijing: Science Press, 2001. 123

16 Fen S Y, Zhang J, Li M J, et al. Organosilicon Polymer and Its Application (in Chinese). Beijing: Chemical Industry Press, 2004. 132

17 Zhang H L, Zhao H L, Jiang M D, et al. How to make a good use of polyacrylamide (in Chinese). Coal Process Compr Util, 2009, 2: 8-14

18 Cao Q F, Song W S, Zhu C C, et al. Summarization on research of di-butyl phthalate. Chin Adhes, 2007, 16: 47-49

19 Zhao Z H. The preparation of underwater self-lubricant bionic coating and its performance analysis (in Chinese). Dissertation for the Master Degree. Harbin: Harbin Engineering University, 2007

20 Walsh M J. Riblets as a viscous drag reduction technique. AIAA J, 1983, 21: 485-486

21 Li G P. Theoretical research of some key problems on oil and gas drag reduction agent and the application and development technology (in Chinese). Dissertation for the Doctoral Degree. Jinan: Shandong University, 2008

Open Access This article is distributed under the terms of the Creative Commons Attribution License which permits any use, distribution, and reproduction in any medium, provided the original author(s) and source are credited. 